\title{
CDISC SDTM Reason Coronary Sinus Access or Left Ventricular Lead Not Implanted Terminology
}

National Cancer Institute

\section{Source}

National Cancer Institute. CDISC SDTM Reason Coronary Sinus Access or Left Ventricular

Lead Not Implanted Terminology. NCI Thesaurus. Code C101864.

Terminology associated with the reason coronary sinus access or left ventricular lead not implanted codelist of the Clinical Data Interchange Standards Consortium (CDISC) Study Data Tabulation Model (SDTM). 